\title{
Evaluation of the validity of the Emotional Regulation Questionnaire in a Mexican sample and their correlation with empathy and alexithymia
}

Victor E. Olalde-Mathieu ${ }^{1}$, Giovanna Licea-Haquet ${ }^{1}$, Azalea Reyes-Aguilar ${ }^{2 *}$, Fernando A. Barrios $^{1 *}$

${ }^{1}$ Universidad Nacional Autónoma de México, Instituto de Neurobiología, Querétaro, México

${ }^{2}$ Universidad Nacional Autónoma de México, Facultad de Psicología, Ciudad de México, México

\section{*Corresponding Authors}

Facultad de Psicología

Universidad Nacional Autónoma de México

Av Universidad 3000, Ciudad de México 04510, Mexico

azaleara@comunidad.unam.mx

Instituto de Neurobiología

Universidad Nacional Autónoma de México

Blvd. Juriquilla 3001, Querétaro, Querétaro, 76230, México

fbarrios@unam.mx

\section{Abstract}

The Emotion Regulation Questionnaire (ERQ) is widely used to measure the individual differences in two emotion regulation strategies: cognitive reappraisal and expressive suppression. In this study, we examine the psychometric properties of the ERQ (Spanish version) in a Mexican community sample $(N=792)$. Exploratory and confirmatory factor analyses demonstrated that the traditional two-factor model (comprising cognitive reappraisal and expressive suppression factors) was replicable and an excellent fit to the data. ERQ cognitive reappraisal $(\alpha=0.81)$ and expressive suppression $(\alpha=0.76)$ scores had acceptable levels of internal consistency reliability. As expected, women tend to use less expressive suppression than men. We also assessed the correlations of both strategies with alexithymia and empathy. Cognitive reappraisal scores were negatively correlated with alexithymia and positively correlated with higher empathy measures, whereas expressive suppression scores were positively correlated with personal distress and alexithymia, and negatively correlated with cognitive empathy 
scales and empathic concern. We conclude that, similar to previous findings, the ERQ has strong psychometric properties in a Mexican community sample and can be used in a confident manner with other tests to complement the assessment of affective traits. In addition, considering previous suggestions of the association between emotional regulation strategies and different components of the empathic response, the correlations between empathy measures and the emotional regulation strategies shown in this study opens a pathway to further research such interactions.

Keywords: Emotion Regulation Questionnaire, emotion regulation, cognitive reappraisal, expressive suppression, alexithymia, empathy 


\section{Introduction}

Emotion regulation plays an essential role in our affective functioning and in our social interactions. Adaptation of our emotional reactions can allow us to adjust poorly matched reactions to situational demands; this can be done by exerting control using a range of strategies. Two of the most studied emotional regulation strategies are cognitive reappraisal and expressive suppression. Cognitive reappraisal consists in attempts to alter the perceive meaning or the intensity of the emotional reaction, by modifying the current appraisal of the situation. On the other hand, expressive suppression refers to the attempt to reduce or inhibit the expressive behavior of the emotion. Both of these strategies were categorized based on the model of emotion regulation proposed by Gross (1998), which states that strategies could be characterized depending on when they exert their effects in the emotional generative process; in this sense cognitive reappraisal and expressive suppression have their effects in different points of the process. Cognitive appraisal acts before the emotion response has been fully charged, altering the emotion trajectory in an efficient manner. Conversely, expressive suppression comes later in the emotion generative process, modifying the behavioral components of the emotion response. The temporal differences between reappraisal and suppression in the emotion generative process imply different effects in psychological functioning in the individual's personal and social life. To assess these differences, Gross and John (2003) developed the Emotional Regulation Questionnaire (ERQ).

The ERQ is a self-report questionnaire that measures the habitual use of reappraisal and suppression. It has two scales with a total of 10 items; 6 of them evaluate cognitive reappraisal (e.g., "When I want to feel less negative emotion, I change what I'm thinking about'), while the other 4 evaluate expressive suppression (e.g., "I control my emotions by not expressing them"). Items are answered with respect to the participant's degree of agreement using a 7-point Likert scale, ranging from 1 (strongly disagree) to 7 (strongly agree). The ERQ presents a two-factorial structure, where reappraisal and suppression 
are two independent regulatory strategies (John \& Gross, 2004), with an adequate internal consistency of $\alpha=0.79$ for cognitive reappraisal and of $\alpha=0.73$ for expressive suppression (Gross \& John, 2003).

Previous research utilizing the ERQ has shown that expressive suppression correlates negatively with age, and has presented sex differences where women tend to use less suppression as a strategy than men. Studies have also shown that the greater use of cognitive reappraisal relates with more beneficial outcomes, such as more social adaptability, lower levels of psychopathology symptoms and better interpersonal functioning (Gross \& John, 2003; Haga et al., 2009; Joormann \& Gotlib, 2010; D. A. Preece et al., 2020). In contrast, greater use of expressive suppression has been related to adverse psychological functioning, greater anxiety and depression, and correlates negatively with the individual's health, this in part because the use of expressive suppression generally causes an increase sympathetic arousal, while augmenting negative affect (Cisler \& Olatunji, 2012; Gross \& John, 2003; Soto et al., 2011). In addition, cognitive reappraisal has been suggested to be correlate negatively with personal distress and alexithymia, whereas expressive suppression has shown to correlate positively (Haga et al., 2009; D. A. Preece et al., 2020). Similarly, reappraisal has been suggested to moderate the empathic response and the relation between affective empathy and prosocial behavior, where greater cognitive reappraisal relates to a lesser association between affective empathy and prosocial behavior; on the other hand, expressive suppression has been suggested to hamper the empathic response, reduce emotion shearing and empathic concern (Butler et al., 2003; Gross \& John, 2003; Lebowitz \& Dovidio, 2015; Lockwood et al., 2014; Naor et al., 2018).

In this study we examine the psychometric properties of the ERQ in a Mexican sample $(N=792)$. We assessed its internal consistency, test-retest reliability, factor structure and relation with other constructs. To assess the further, we evaluated the correlation of cognitive reappraisal and expressive suppression, with established measures of alexithymia (20-item Toronto Alexithymia Scale [TAS-20]; Bagby et al., 1994; Moral de la Rubia, J., 2008) and cognitive and affective empathy (Interpersonal 
Reactivity Index [IRI]; Davis, 1980; Velázquez et al., 2019). Alexithymia has been related with reappraisal and suppression strategies (D. Preece et al., 2018; D. A. Preece et al., 2020), people with alexithymia have difficulties processing their emotions resulting in a reduced emotional awareness, which appears to be a crucial rate-limiting factor in successfully regulating emotions (Gross, 2015). Emotional regulation also plays an important role modulating the empathic response, reappraisal and suppression strategies interact with both affective and cognitive aspects of empathy (Decety, 2011; Lockwood et al., 2014; Zaki \& Ochsner, 2012).

Given other evaluations of the ERQ in Spanish speaking countries we expect adequate internal consistency scores and that the two-factor structure will be maintain in the Mexican sample (Pérez \& Bello, 2017). Also given the previous research we expect that the psychometric properties of the ERQ concord with those shown in other countries, including sex differences and age correlation. We also expect to find the predicted correlations with other constructs, cognitive reappraisal will correlate negatively with alexithymia scores and positively with greater empathic scores, while expressive suppression will correlate positively with alexithymia scores and negatively with greater empathic scores.

\section{Methods}

\section{Participants}

A total of 792 (55\% females) Mexican participants, ranging in age from 17 to 76 (mean $=27.1$, sd=11.7), concluded the Spanish version of the ERQ, 699 (56\% females) of them also completed the TAS-20 and IRI, to assess alexithymia and cognitive and affective empathy. A subset of 101 participants (61\% females), ranging in age from 17 to 62 (mean=34.0, sd=9.7), completed the ERQ 
after 3 months for test-retest reliability. All participants were native Spanish speakers, all of them were volunteers and receive nothing for their participation.

\section{Measures}

The administered questionnaires where the ERQ (Gross \& John, 2003; Rodríguez-Carvajal et al., 2006), TAS-20 (Bagby et al., 1994; Moral de la Rubia, J., 2008) and the IRI (Davis, 1980; Velázquez et al., 2019). The Emotional Regulation Questionnaire Spanish version is a 10 item self-report questionnaire, consisting of two scales where each measures the habitual use of an emotional regulation strategy: cognitive reappraisal (6 items) and expressive suppression (4 items). The items are responded within a 7 point Likert ( $1=$ strongly disagree, $7=$ strongly agree $)$.

Toronto Alexithymia Scale. Is a 20 item self-report questionnaire, designed to measure alexithymia within three subscales: difficulty identifying one's own feelings (DIF; 7 items), difficulty describing feelings (DDF; 5 items) and an externally orientated thinking style (EOT; 8 items), the items are responded in a 5 point Likert scale $(1=$ strongly disagree, $5=$ strongly agree $)$. All items are summed into a total alexithymia score. Higher scores indicate higher levels of alexithymia.

Interpersonal Reactivity Index. Is a 28 item self-report questionnaire, which responses are measured on a 5-point Likert-type scale ranging from 4 (describes me very well) to 0 (does not describe me well). The IRI measures cognitive and affective empathy within four independent scales: Fantasy (FS), Perspective Taking (PT), Empathic Concern (EC), and Personal Distress (PD). Each of the four subscales are comprised by 7 items, with subscales scored as the sum of the items. Greater scores in fantasy, perspective taking and empathy concern, indicate greater empathic abilities, while lower scores in personal distress indicate greater empathy (M. H. Davis, 1983).

\section{Data Analyses}


All statistical analysis were done using R software (R Core Team, 2019), using in-house scripts and packages psych and lavaan (William Revelle, 2018; Yves Rosseel, 2012). Psychometric properties of the ERQ were evaluated, means, standard deviations, Cronbach's $\alpha$ reliability coefficient and testretest reliability coefficients were obtained. Univariate and multivariate preliminary distribution analysis was indicative of non-normality. For the test-retest reliability we used interclass correlation coefficients (ICC). To assess the two-factor model, the Satorra-Bentler scaled correction of maximumlikelihood was used for the confirmatory factor analysis (CFA), since it provides a more robust fit (Hu L. T. et al., 1992). As reported in Gross and John (Gross \& John, 2003) we compared, three CFA models: a) general-factor model, b) hierarchical model, which posits that some individuals regulate more using both strategies and some individuals regulate less using neither, and c) independence model, were the factor correlation was set to 0 . The fit of the models was compared statistically with the least parsimonious or augmented model, a two-factor model were the factor intercorrelation was freely estimated. To evaluate differences between sexes and correlations with other constructs, we converted to z-values, applied a t-test controlling age as a covariate, and for the correlations, we did partial correlations controlling sex as a covariate. A Pearson and Filon's comparison test was performed to evaluate differences in the magnitude of two correlations (Diedenhofen \& Musch, 2015), contrasting the correlation of cognitive reappraisal with a given construct, with the correlation of expressive suppression with the same construct.

\section{Results}

\section{Psychometric properties and factor analysis}


The internal consistency measure by Cronbach's $\alpha$ coefficients, was $\alpha=0.80$ for cognitive reappraisal and $\alpha=0.76$ for expressive suppression. For the test-retest reliability, the ICC was $0.81(\mathrm{p}<0.001)$ for reappraisal and $0.82(\mathrm{p}<0.001)$ for suppression.

Similar to Gross and John (Gross \& John, 2003), across all the standard fit indexes the independence model and the augmented model, presented the better fit. The goodness of fit indices for the independence model were, $\mathrm{S}-\mathrm{B} \chi_{2}(35)=251.35(\mathrm{p}<0.001), \mathrm{CFI}=0.90, \mathrm{RMSEA}=0.09, \mathrm{SRMR}=0.08$; and for the augmented model were, $\mathrm{S}-\mathrm{B} \chi_{2}(34)=239.50(\mathrm{p}<0.001), \mathrm{CFI}=0.90, \mathrm{RMSEA}=0.09$, $\mathrm{SRMR}=0.07$. The zero order correlation between the factors was $\mathrm{r}=0.12(\mathrm{p}<0.001)$. CFA standardized factor loadings for both models can be seen in table1. Although both models presented a good fit, the augmented model showed a better fit when compared to the independence model, $\Delta \chi^{2}(1)$ $=12.83(\mathrm{p}<0.001)$.

Age correlated negatively with expressive suppression $(r=-0.17, \mathrm{p}<0.001)$ but no with cognitive reappraisal. Sex differences, were only found in the use of expressive suppression $t=3.81(\mathrm{p}<0.001)$, with a Cohens'd of 0.25 , were woman (mean=3.37, $\mathrm{sd}=1.4)$ suppress less than man (mean=3.71, $\mathrm{sd}=$ $1.3)$.

\section{Correlation with other constructs}

As expected, cognitive reappraisal correlated positively with higher empathy scores, in both cognitive empathy measures (FS, PT) and empathic concern, while suppression correlated negatively with the same constructs with the exception of FS. In addition, cognitive reappraisal correlated negatively with personal distress and all the alexithymia scales, while suppression correlated positively with the same constructs (Table2). Taking into account that both strategies correlated with almost all of the same constructs, we next compared the magnitude of such correlations between the strategies and their respective constructs (Table3). Cognitive reappraisal showed stronger positive correlations with three 
empathy subscales, FS, PT and EC, while expressive suppression had stronger positive correlations with the other set of measures such as PD, DDF, DIF, and EOT. 
Table 1. Confirmatory standardized factor loadings of both augmented and independence models, for reappraisal and suppression items.

Scale/item number. Items in English and Spanish translation

Augmented

Independence

Cognitive

reappraisal

1.- When I want to feel more positive emotion (such as joy or amusement), I change what I'm thinking about.

Cuando quiero sentir más una emoción positiva (por ejemplo, alegre o divertido), modifico lo que pienso sobre ello.

3.- When I want to feel less negative emotion (such as sadness or anger), I change what I'm thinking about.

Cuando quiero sentir menos una emoción negativa (por ejemplo, tristeza o enfado), modifico lo que pienso sobre ello.

5.- When I'm faced with a stressful situation, I make myself think about it in a way that helps me stay calm.

Cuando hago frente a una situación estresante, pienso en ella de una manera que me ayude a mantener la calma.

7.- When I want to feel more positive emotion, I change the way I'm thinking about the situation.

Cuando quiero sentir más una emoción positiva, cambio la manera de pensar sobre esa situación.

8.- I control my emotions by changing the way I think about the situation I'm in.

Controlo mis emociones cambiando la manera de pensar sobre la situación en la que me encuentro.

10.- When I want to feel less negative emotion, I change the way I'm thinking about the situation.

Cuando quiero sentir menos una emoción negativa, cambio la manera de pensar sobre esa situación. Expressive suppression

2.- I keep my emotions to myself.

Trato de mantener ocultos mis sentimientos.

4.- When I am feeling positive emotions, I am careful not to express them.

Cuando estoy sintiendo emociones positivas, tengo cuidado de no expresarlas.

6.- I control my emotions by not expressing them.

Controlo mis emociones no expresándolas.

9.- When I am feeling negative emotions, I make sure not to express them.

Cuando estoy sintiendo emociones negativas, me aseguro de no expresarlas.

Notes. $\mathrm{N}=792$. All factor loadings were statistically significant $(\mathrm{p}<0.001)$ 
Table2. Relations with other constructs.

\begin{tabular}{lcccc}
\hline & Construct & rho & p.value* & t.value \\
\hline Cognitive Reappraisal & & & & \\
& FS & 0.10 & $\mathbf{0 . 0 1 2}$ & 2.605 \\
& PT & 0.24 & $>\mathbf{0 . 0 0 1}$ & 6.394 \\
& EC & 0.12 & $\mathbf{0 . 0 0 2}$ & 3.239 \\
& PD & -0.09 & $\mathbf{0 . 0 2 1}$ & -2.344 \\
& DDF & -0.11 & $\mathbf{0 . 0 0 4}$ & -2.99 \\
& DIF & -0.09 & $\mathbf{0 . 0 1 9}$ & -2.395 \\
& EOT & -0.15 & $>\mathbf{0 . 0 0 1}$ & -3.892 \\
& TAS total & -0.14 & $>\mathbf{0 . 0 0 1}$ & -3.658 \\
& & & & \\
& FS & 0.01 & 0.887 & 0.1417 \\
& PT & -0.14 & $>\mathbf{0 . 0 0 1}$ & -3.697 \\
& EC & -0.20 & $>\mathbf{0 . 0 0 1}$ & -5.315 \\
& PD & 0.12 & $\mathbf{0 . 0 0 3}$ & 3.066 \\
& DDF & 0.47 & $\mathbf{> 0 . 0 0 1}$ & 14.13 \\
& DIF & 0.37 & $>\mathbf{0 . 0 0 1}$ & 10.35 \\
& EOT & 0.26 & $>\mathbf{0 . 0 0 1}$ & 7.112 \\
& TAS total & 0.45 & $>\mathbf{0 . 0 0 1}$ & 13.14 \\
\hline
\end{tabular}

Results are shown controlling Sex and Age as covariates Interpersonal reactivity index (IRI) scales: $\mathrm{PT}=$ Perspective Taking, FS=Fantasy, $\mathrm{EC}=$ Empathy Concern, $\mathrm{PD}=$ Personal Distress. Toronto Alexithymia Scale (TAS-20) scales: DDF=difficulty describing feelings, DIF=difficulty identifying feelings, EOT= externally orientated thinking. *FDR-

corrected.

Table 3. Pearson and Filon's z correlation differences

\begin{tabular}{lclccc}
\hline $\begin{array}{l}\text { Reappraisal } \\
\text { correlations }\end{array}$ & rho & $\begin{array}{l}\text { Suppression } \\
\text { correlations }\end{array}$ & rho & z-value & p.value \\
\hline Reap-FS & $\mathbf{0 . 0 9 8 4 *}$ & Supre-FS & 0.00538 & 2.0 & 0.043 \\
Reap-PT & $\mathbf{0 . 2 3 5 9 *}$ & Supre-PT & -0.139 & 8.6 & $>0.001$ \\
Reap-EC & $\mathbf{0 . 1 2 2} *$ & Supre-EC & -0.1978 & 7.7 & $>0.001$ \\
Reap-PD & -0.08862 & Supre-PD & $\mathbf{0 . 1 1 5 6 *}$ & -3.6 & $>0.001$ \\
Reap-DDF & -0.1128 & Supre-DDF & $\mathbf{0 . 4 7 2 7 *}$ & -13.4 & $>0.001$ \\
Reap-DIF & -0.09052 & Supre-DIF & $\mathbf{0 . 3 6 5 6 *}$ & -10.3 & $>0.001$ \\
Reap-EOT & -0.1461 & Supre-EOT & $\mathbf{0 . 2 6 0 6 *}$ & -9.7 & $>0.001$ \\
Reap-TAS_Tot & -0.1375 & Supre-TAS_Tot & $\mathbf{0 . 4 4 6 5 *}$ & -13.6 & $>0.001$ \\
\hline
\end{tabular}

*Statistically significant stronger correlation. Reap=Reappraissal,

Supre=Suppression. Interpersonal reactivity index (IRI) scales: PT=Perspective

Taking, FS=Fantasy, EC=Empathy Concern, $\mathrm{PD}=$ Personal Distress. Toronto

Alexithymia Scale (TAS-20) scales: DDF=difficulty describing feelings,

$\mathrm{DIF}=$ difficulty identifying feelings, EOT = externally orientated thinking. 


\section{Discussion}

The results confirmed the reliability and two-factor structure of the ERQ Spanish version in a Mexican sample. The reliability and internal consistency coefficients of reappraisal and suppression were comparable to those obtained in the original version and in other samples (Balzarotti et al., 2010; Cabello et al., 2013; Gross \& John, 2003; D. A. Preece et al., 2020). Testretest reliability supported a temporal stability of the ERQ, while CFA corroborated the two-factor structure presented in Gross and John (2003). Although, the independence model statistically differs from the augmented model, the fitness of the independent model was still a good fit in all parameters and performed better than the other tested models. This could mean that, although the relationship between factors is greater than in other samples, still its performance is more incline to an independence model, were individuals that frequently use reappraisal strategy are no more/less likely to use suppression; this inclination is also supported by the lesser fit of the propose interactions suggested by other models.

The performance of the ERQ was also similar to other samples, in respect to the predicted correlation between age and expressive suppression, where the greater the age the less use of expressive suppression. The sex differences were also as expected, none present in cognitive reappraisal, whereas in expressive suppression, woman tend to suppress less than man. Similarly, the expected correlations between the emotional regulation strategies and, alexithymia and empathy (Lockwood et al., 2014; D. A. Preece et al., 2020), were also present in this study. Cognitive reappraisal correlated negatively with alexithymia and positively with higher empathic measures. Cognitive reappraisal has been related to positive affect, being considered as a good adaptive regulation strategy, given that alexithymia is a limiting factor for a successful emotion regulation, the relation between this two constructs has been considered to be negative, as shown 
in this study. Reappraisal has also been related to higher scores in affective and cognitive empathy, this relation was also portrait in this study, positively with the two cognitive empathy scales (fantasy, perspective taking) and with the affective empathy scale of empathic concern, and negatively with personal distress, which is in concordance with the negative relation between personal distress and the other scales (M. H. Davis, 1983). Contrary to cognitive reappraisal, suppression has been generally related with maladaptive behavior and enhancing negative affect, hence that expressive suppression presented the same relation, but in an opposite manner, with all the scales, with the exception of the fantasy scale. The relation of both strategies with both positive and negative affect, was further reflected in the differences of the magnitudes of their respective correlations with other constructs, as shown by the Pearson and Filon's z test, were cognitive reappraisal correlate more strongly with those constructs that had a positive direction towards positive affect, and expressive suppression correlated more strongly with those that had a negative direction towards negative affect.

Our findings suggest that the ERQ can be used confidently, with the complete 10-item version and a two-factor structure, in Mexican samples. The relationships between ERQ, with the TAS-20 and IRI, could open a path to further investigate the relationship between emotional regulation strategies, positive behavior and affective personality traits.

\section{Acknowledgements}

Víctor Enrique Olalde Mathieu is a doctoral student from "Programa de Doctorado en Ciencias Biomédicas", Universidad Nacional Autónoma de México (UNAM) and has recived CONACyT fellowship no. 330989 (No.CVU: 619655). This work was supported by grants from DGAPA- 
PAPIIT UNAM grant IN202119 (FAB) and CONACyT grant CB255462 (FAB). We thank Nuri Aranda López, Leonor Casanova Rico, Leopoldo González-Santos, Ma. de Lourdes Lara Ayala, Juan J. Ortiz and Erick H. Pasaye for their technical support. M.C. Jeziorski for editing of the manuscript.

\section{Competing interests}

The authors declare no competing interest. 


\section{References}

Bagby, R. M., Parker, J. D. A., \& Taylor, G. J. (1994). The twenty-item Toronto Alexithymia scale-I. Item selection and cross-validation of the factor structure. Journal of Psychosomatic Research, 38(1), 23-32. https://doi.org/10.1016/0022-3999(94)90005-1

Balzarotti, S., John, O. P., \& Gross, J. J. (2010). An Italian Adaptation of the Emotion Regulation Questionnaire. European Journal of Psychological Assessment, 26(1), 61-67. https://doi.org/10.1027/1015-5759/a000009

Butler, E. A., Egloff, B., Wlhelm, F. H., Smith, N. C., Erickson, E. A., \& Gross, J. J. (2003). The social consequences of expressive suppression. Emotion, 3(1), 48-67. https://doi.org/10.1037/1528-3542.3.1.48

Cabello, R., Salguero, J. M., Fernández-Berrocal, P., \& Gross, J. J. (2013). A Spanish Adaptation of the Emotion Regulation Questionnaire. European Journal of Psychological Assessment, 29(4), 234-240. https://doi.org/10.1027/1015-5759/a000150

Cisler, J. M., \& Olatunji, B. O. (2012). Emotion Regulation and Anxiety Disorders. Current Psychiatry Reports, 14(3), 182-187. https://doi.org/10.1007/s11920-012-0262-2

Davis, M. (1980). A multidimensional approach to individual differences in empathy. JSAS Catalog of Selected Documents in Psychology, 10(85).

Davis, M. H. (1983). Measuring individual differences in empathy: Evidence for a multidimensional approach. Journal of Personality and Social Psychology, 44(1), 113126. https://doi.org/10.1037/0022-3514.44.1.113

Decety, J. (2011). Dissecting the Neural Mechanisms Mediating Empathy. Emotion Review, 3(1), 92-108. https://doi.org/10.1177/1754073910374662 
Diedenhofen, B., \& Musch, J. (2015). cocor: A Comprehensive Solution for the Statistical Comparison of Correlations. PLoS ONE, 10(4). https://doi.org/10.1371/journal.pone.0121945

Gross, J. J. (1998). The emerging field of emotion regulation: An integrative review. Review of General Psychology, 2(3), 271-299. https://doi.org/10.1037/1089-2680.2.3.271

Gross, J. J. (Ed.). (2015). Handbook of Emotion Regulation, Second Edition (Second edition). The Guilford Press.

Gross, J. J., \& John, O. P. (2003). Individual differences in two emotion regulation processes: Implications for affect, relationships, and well-being. Journal of Personality and Social Psychology, 85(2), 348-362. https://doi.org/10.1037/0022-3514.85.2.348

Haga, S. M., Kraft, P., \& Corby, E.-K. (2009). Emotion Regulation: Antecedents and Well-Being Outcomes of Cognitive Reappraisal and Expressive Suppression in Cross-Cultural Samples. Journal of Happiness Studies, 10(3), 271-291. https://doi.org/10.1007/s10902007-9080-3

Hu L. T., Bentler P. M., \& Kano Y. (1992). Can test statistics in covariance structure analysis be trusted? 112(2), 351.

John, O. P., \& Gross, J. J. (2004). Healthy and Unhealthy Emotion Regulation: Personality Processes, Individual Differences, and Life Span Development. Journal of Personality, 72(6), 1301-1334. https://doi.org/10.1111/j.1467-6494.2004.00298.x

Joormann, J., \& Gotlib, I. H. (2010). Emotion regulation in depression: Relation to cognitive inhibition. Cognition and Emotion, 24(2), 281-298.

https://doi.org/10.1080/02699930903407948 
Lebowitz, M. S., \& Dovidio, J. F. (2015). Implications of emotion regulation strategies for empathic concern, social attitudes, and helping behavior. Emotion, 15(2), 187-194. https://doi.org/10.1037/a0038820

Lockwood, P. L., Seara-Cardoso, A., \& Viding, E. (2014). Emotion Regulation Moderates the Association between Empathy and Prosocial Behavior. PLoS ONE, 9(5), e96555. https://doi.org/10.1371/journal.pone.0096555

Moral de la Rubia, J. (2008). Propiedades psicométricas de la Escala de Alexitimia de Toronto de 20 reactivos en México. Revista Electrónica de Psicología Iztacala, 11(2).

Naor, N., Shamay-Tsoory, S. G., Sheppes, G., \& Okon-Singer, H. (2018). The impact of empathy and reappraisal on emotional intensity recognition. Cognition and Emotion, 32(5), 972-987. https://doi.org/10.1080/02699931.2017.1372366

Pérez, O. I. G., \& Bello, N. C. (2017). Regulación emocional: Escalas de medición en español [revisión psicométrica]. Revista Iberoamericana de Psicología, 10(2), 81-91. https://doi.org/10.33881/2027-1786.rip.10209

Preece, D. A., Becerra, R., Robinson, K., \& Gross, J. J. (2020). The Emotion Regulation Questionnaire: Psychometric Properties in General Community Samples. Journal of Personality Assessment, 102(3), 348-356.

https://doi.org/10.1080/00223891.2018.1564319

Preece, D., Becerra, R., Robinson, K., \& Dandy, J. (2018). Assessing Alexithymia: Psychometric Properties and Factorial Invariance of the 20-Item Toronto Alexithymia Scale in Nonclinical and Psychiatric Samples. Journal of Psychopathology and Behavioral Assessment, 40(2), 276-287. https://doi.org/10.1007/s10862-017-9634-6 
R Core Team, R. C. (2019). R : A language and environment for statistical computing. Http://Www.R-Project.Org. https://ci.nii.ac.jp/naid/20001689445/

Rodríguez-Carvajal, R., Moreno-Jiménez, B., \& Garrosa, E. (2006). Cuestionario de regulación emocional. Versión Española. Autorizado Por Los Autores de La Versión Original En Inglés (Gross \& John, 2003).

Soto, J. A., Perez, C. R., Kim, Y.-H., Lee, E. A., \& Minnick, M. R. (2011). Is expressive suppression always associated with poorer psychological functioning? A cross-cultural comparison between European Americans and Hong Kong Chinese. Emotion, 11(6), 1450-1455. https://doi.org/10.1037/a0023340

Velázquez, E. S. M., Moreno, A. V., Aguilar, G. G., \& González, A. L. A. (2019). Propiedades psicométricas del Interpersonal Reactivity Index (IRI) en mexicanos universitarios. Revista Iberoamericana de Psicología: Ciencia y Tecnología, 12(1), 111-122.

William Revelle. (2018). psych: Procedures for Psychological, Psychometric, and Personality Research. Northwestern University. https://CRAN.R-project.org/package=psych

Yves Rosseel. (2012). lavaan: An R Package for Structural Equation Modeling. Journal of Statistical Software, 48(2), 1-36.

Zaki, J., \& Ochsner, K. N. (2012). The neuroscience of empathy: progress, pitfalls and promise. Nature Neuroscience, 15(5), 675-680. https://doi.org/10.1038/nn.3085 